amplifying pre-existing ones.

The melanoma drug

ipilimumab boosts the activity of immune-system cells called $T$ cells, which recognize a variety of molecules on tumour cells, marking them for destruction. Pia Kvistborg and Ton Schumacher of the Netherlands Cancer Institute in Amsterdam and their team compared $\mathrm{T}$ cells from melanoma patients before and after treatment and found that the drug broadened the range of melanoma targets recognized by T cells. But melanoma-specific $\mathrm{T}$ cells that were already present before treatment did not expand their ranks, suggesting that the drug stimulates new responses.

Boosting the range of $\mathrm{T}$ cells could be a useful strategy for other cancer immunotherapies, the authors say.

Sci. Transl. Med. 6, 254ra128 (2014)

\section{QUANTUM OPTICS}

\section{Photons made to dance together}

Physicists have made two beams of light interact at the level of individual photons.

Getting photons to interact is important for all-optical computation and for producing new quantum states of light. Kristin Beck at the Massachusetts Institute of Technology in Cambridge and her colleagues crossed two beams of light inside a cavity filled with trapped and cooled caesium atoms. When photons from each beam tried to pass through the system at the same time, the trapped atoms changed their internal state, allowing only one photon to be transmitted, while the other one was reflected or scattered.

The interaction creates two entangled beams of light, which the authors say could eventually be used to improve the accuracy of measurements, such as of a gyroscope's rotation, that would otherwise be limited by the laws of quantum mechanics.

Phys. Rev. Lett. 113, 113603

(2014)
CHEMISTRY

\section{On the trail of a drug in plants}

The painkiller tramadol is not made naturally by plants despite last year's surprising finding that the drug was present in the roots of a Cameroonian plant (Nauclea latifolia).

Michael Spiteller from the Technical University of Dortmund in Germany and his team analysed plant, water and soil samples in Cameroon and interviewed local residents to track down the true explanation. Farmers in the far north area have been giving tramadol to their cattle, which then excrete the drug and its metabolites into soil and water. The painkiller is subsequently absorbed by plant roots.

The authors say this offlabel use of tramadol poses a risk to health because it also contaminates local water supplies.

Angew. Chem. Int. Ed. http://doi. org/f2t7tg (2014)

\section{GENOMICS \\ Mutation has big disease effect}

A genetic mutation is associated with a change in a person's risk of heart disease from high to low - one of the first rare gene variants found, by sequencing large numbers of people, that has a sizable impact on disease risk.

Nicole Soranzo of the Wellcome Trust Sanger Institute in Hinxton and Nicholas Timpson of the University of Bristol, both in the United Kingdom, led a team that sequenced the genomes of 3,202 Europeans with known levels of blood triglycerides - fatty acids linked to increased risk of heart disease. They discovered that one variant of the APOC3 gene, found in less than $0.2 \%$ of the study participants, was associated with reductions in triglyceride levels that they estimated were large enough to significantly lower an

SOCIAL SELECTION Romituraticat

\title{
How to draw perfect figures
}

Jumbled charts and misleading graphs - illustrations in a paper can go wrong in many ways. Now, a treatise that attempts to rescue science from bad figures has been getting rave reviews on social media.

Using original illustrations - some elegant, some clunky - to prove key points, 'Ten simple rules for better figures' tries to steer researchers away from common pitfalls. The first tip: know your audience. A stripped-down graph that might make sense to your closest colleagues could prove baffling to anyone else. Many researchers on social media were grateful for the advice. Andrew Jackson, an evolutionary ecologist at Trinity College Dublin, tweeted: "Magic. Using that in lectures for sure. Incoming students take note!" PLoS Comput. Biol. 10, e1003833 (2014)

\begin{tabular}{l|l}
\hline $\begin{array}{l}\text { Based on data from altmetric.com. } \\
\text { Altmetric is supported by Macmillan }\end{array}$ & $\begin{array}{l}\text { For more on } \\
\text { Science and Education, which owns } \\
\text { Nature Publishing Group. }\end{array}$ \\
\hline
\end{tabular}

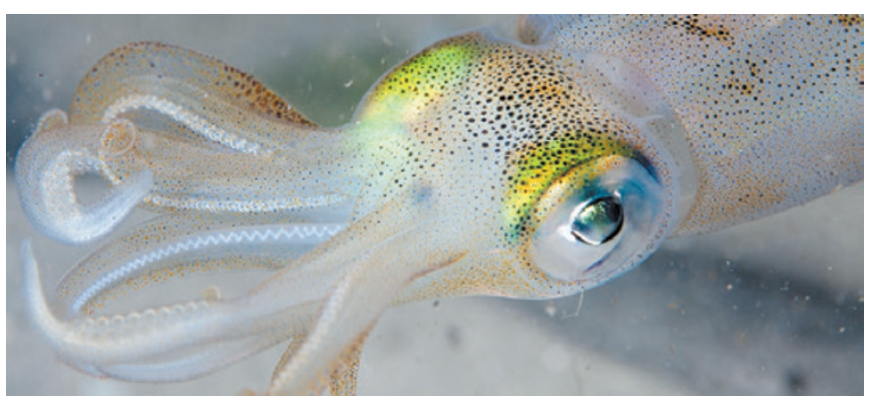

individual's risk level.

The results support the idea that sequencing large populations can uncover rare genetic mutations that have a major impact on disease risk. Nature Commun. 5, 4871 (2014)

\section{MATERIALS}

\section{Squid skin inspires colourful display}

A flexible material can display patterns much in the same way that cuttlefish, octopuses and squid (pictured) form colourful spots in their skin.

To quickly change their appearance and camouflage themselves, such cephalopods use their muscles to stretch and relax small sacs of pigment under their skin. A team led by Xuanhe
Zhao of the Massachusetts Institute of Technology in Cambridge developed an artificial equivalent using silicone rubber and organic compounds called spiropyran mechanophores, which glow when exposed to force. The researchers control the display by applying an electric field to deform the silicone, causing the mechanophores to glow in various repeated patterns including lines, circles and letters.

The display size and resolution could be scaled up for use as a camouflaging coating, the authors say.

Nature Commun. 5, 4899 (2014)

\section{DNATURE.COM}

For the latest research published by Naturevisit:

www.nature.com/latestresearch 